\title{
Single incision laparoscopic adrenalectomy - initial experience
}

\author{
Marcin Łosin' ${ }^{1}$, Piotr Czauderna ${ }^{1}$, Andrzej Gołębiewski1, Joanna Stefanowicz² \\ 1Department of Surgery and Urology for Children and Adolescents, Medical University of Gdansk, Poland \\ 2Department of Paediatrics, Haematology, Oncology and Endocrinology, Medical University of Gdansk, Poland
}

Videosurgery and other miniinvasive techniques 2010; 5 (3): 104-106 DOI: 10.5114/wiitm.2010.16421

\begin{abstract}
A laparoscopic approach to the adrenal gland was introduced in 1992. Since then many studies on adult populations evaluating the safety and results of laparoscopic surgery have been published. A laparoscopic approach to adrenal surgery in children still remains challenging. During the last 20 years we have observed a tendency to minimize surgical trauma that challenged many surgeons to seek new approaches in laparoscopic surgery. Single incision laparoscopic surgery (SILS), in which all instruments are placed through a single, small incision, might be a step towards less invasive surgical procedures. We present two cases of paediatric patients treated in our department with single incision laparoscopic adrenalectomy (SILA). According to our best knowledge this report includes the first such procedure performed in Poland.
\end{abstract}

Key words: adrenalectomy, laparoscopy, SILS, paediatric surgery

\section{Introduction}

A laparoscopic approach to the adrenal gland was introduced in 1992. Since then many studies on adult populations evaluating the safety and results of laparoscopic surgery have been published. A laparoscopic approach to adrenal surgery in children still remains challenging, mostly because tumours in the paediatric population are usually bigger than in adults and all dissection has to be done in a relatively small area. A few papers have shown that laparoscopy is a feasible and safe approach to adrenalectomy in the paediatric population. During the last 20 years we have observed a tendency to minimize surgical trauma that challenged many surgeons to seek new approaches in laparoscopic surgery. Single incision laparoscopic surgery (SILS), in which all instruments are placed through a single, small incision, might be a step towards less invasive surgical procedures. One of the major goals of introducing laparoscopic surgery was to reduce postoper- ative pain and improve the cosmetic result, so SILS may be considered as the next step to virtually "scarless" surgery. We present two cases of paediatric patients treated in our department with single incision laparoscopic adrenalectomy (SILA). According to our best knowledge this report includes the first such procedure performed in Poland.

\section{Material and methods}

Between December 2009 and January 2010 we performed single incision adrenalectomy in two children. This technique was modified in the first case, who was an 11-month old boy suffering from disseminated neuroblastoma (NB) localized in both adrenal glands. Metastases to the liver and lungs were detected, but no amplification of N-myc was found. Primary adrenal tumours were almost $4 \mathrm{~cm}$ in diameter, each. Open biopsy of one of the liver metastatic lesions revealed NB Schwannian stroma, poorly differentiated. The child received chemotherapy con- 
sisting of VP-16 and CARBO (etoposide, carboplatin) and after 2 courses a mixed response was observed; thus chemotherapy was continued with two additional courses. Then after adrenal tumour shrinkage to less than $2 \mathrm{~cm}$ and after achieving stabilization of pulmonary and liver metastases, two additional CADO courses were administered. Next, the patient was scheduled for surgery. In the supine position a 2-cm long longitudinal incision was made at the umbilicus and the abdominal cavity was opened, then the Covidien SILS Port was introduced. The abdominal cavity was inflated with carbon dioxide up to a pressure of $12 \mathrm{mmHg}$. After abdominal inspection an additional 3-mm port was introduced percutaneously in the middle, just underneath the xiphoid process, to retract the liver. To dissect the right adrenal gland with tumour from surrounding organs, a monopolar hook and harmonic scalpel were used. A single articulated instrument was used (Covidien Roticulator), too. Right adrenal vessels were clipped and cut, then the adrenal gland together with the tumour was removed completely. No enlargement of local lymph nodes was observed. Then the splenic flexure of the transverse colon was mobilized and the upper left renal pole was exposed. Due to problems with visualization of the left adrenal gland we decided to perform whole mobilization of the spleen. Also percutaneously introduction of an additional 3-mm grasper to retract the stomach was required. One-centimetre tumour was localized in the peripheral part of the left adrenal gland. After its exposure partial adrenalectomy was performed with a harmonic scalpel. No pathological lesion in the liver was observed, since no biopsy was performed. Both adrenal tumours were removed in an Endo-catch sac (Covidien) through the umbilical incision. Pathological examination confirmed the diagnosis of neuroblastoma and showed clear resection margins. Operating time was 220 min and there were no postoperative complications, except for minor infection of the umbilicus, which was treated conservatively and resolved after a few days. The child continued chemotherapy with etoposide and carboplatin and remains free of disease at the 4th month of follow-up from completion of the treatment.

The second case was a 15-year-old girl suffering from a left adrenal tumour. She was admitted to the hospital due to abdominal pain. After revealing an abdominal mass in the ultrasound examination, abdominal CT confirmed the presence of a $4-\mathrm{cm}$ tumour in the left adrenal gland. No typical malignancy signs were observed, so the child underwent surgery without initial chemotherapy. We decided to use the SILA approach. The child was placed in the right lateral position, and a Covidien SILS Port was introduced in the left epigastrium, lateral to the rectus muscle. The abdominal cavity was inflated with $\mathrm{CO}_{2}$, then the splenic flexure of the transverse colon and the spleen together with the caudal part of the pancreas were mobilized with a LigaSure device (Covidien), monopolar hook and special articulated instruments (Roticulator by Covidien). The upper renal pole was exposed and a $4-\mathrm{cm}$ wide tumour mass was identified in the adrenal gland near to the renal hilum. Adrenal vessels were clipped and cut, then the tumour in continuity with the left adrenal gland was dissected. No enlargement of local lymph nodes was observed. The specimen was removed in an Endo-catch sac (Covidien). Operating time was $135 \mathrm{~min}$ and no postoperative complications were observed. Microscopic examination revealed benign ganglioneuroma. Follow-up is 2 months and there are no signs of local recurrence.

\section{Discussion}

Laparoscopic adrenalectomy was introduced in 1992 by Gagner et al. [1] and it has become an interesting alternative to open surgery ever since and the procedure of choice for surgical management of most adrenal tumours. Many patients have undergone this procedure with good results and many studies have shown the benefit of laparoscopic surgery [2, 3]. The majority of papers describe adult populations with small benign adrenal tumours [4, 5]. The role of laparoscopic adrenalectomy in malignant adrenal tumours still remains controversial; however there are some reports on this approach in paediatrics [5, 7]. The results of laparoscopic adrenalectomies in neuroblastoma (LA) are very encouraging since the final outcome is comparable to open surgery and LA remains a safe procedure [8]. Achieving a complete macroscopic resection in over $95 \%$ of a series of 47 children has been reported [7].

In our department we have performed laparoscopic adrenalectomy in neuroblastoma since 2006 with good results and we have proven that this is a safe and feasible approach in selected cases. Laparoscopic adrenal surgery provides the same or even better visualization as an open technique, 
enables safe dissection of the adrenal gland and improves the cosmetic result [9]. This might be an even more interesting technique in small adrenal tumour cases which require a large incision to expose the retroperitoneal space in open surgery. Evolution of new techniques, such as robotic surgery, shows the demand for new approaches in surgery. One of the very visible trends is to minimize surgical trauma and there is an obvious need among patients for "scarless" surgery. Single incision laparoscopic surgery, in which all instruments are placed through a single, small incision, might be a step toward even less invasive surgical procedures. A few authors have reported successful use of SILS procedures in a paediatric population, including SILS cholecystectomy, splenectomy and appendectomy [10, 11]. We decided to perform SILS adrenalectomy in two patients with adrenal tumours. One was neuroblastoma and the other ganglioneuroma. Both procedures were feasible and safe. However, there might be some limitations of this approach. In particular, the need to use special articulated instruments, lack of instrument triangulation and operating partly with the left hand make SILS more demanding. On the other hand, there is always a possibility to modify the technique by using additional percutaneously inserted 3-mm instruments (as in case No. 1) or to convert to standard laparoscopy or open technique. In our cases the operating time was similar to standard laparoscopic adrenalectomy and there were no major postoperative complications recorded.

We understand that this is a very small series and we cannot make any definite conclusion but the early experience with SILS adrenalectomy in children appears to be encouraging and effective. However, further studies and greater numbers will be required to investigate the potential benefits of this approach.

\section{References}

1. Gagner M, Lacroix A, Bolte E. Laparoscopic adrenalectomy in Cushing's syndrome and pheochromocytoma. N Engl J Med 1992; 327: 1033

2. Gagner M, Pomp A, Heinford BT, et al. Laparoscopic adrenalectomy: lessons learned from 100 consecutives procedures. Ann Surg 1997; 226: 238-46.

3. Zeth HJ, Udelsman R. One hundred laparoscopic adrenalectomies: a single surgeon's experience. Ann Surg Oncol 2003; 10 1012-7.

4. O'Boyle C, Kapadia CR, Sedman PC, et al. Laparoscopic transperitoneal adrenalectomy. Surg Endosc 2003; 17: 1905-9.

5. Toutounchi S, Cieśla W, Krajewska E, et al. Laparoscopic enucleation of a single adrenal tumour in a patient suffering from pri- mary hyperaldosteronism. Videosurgery and other miniinvasive techniques 2007; 2: 164-7.

6. De Lagausie, Berrebi D, Michon J, et al. Laparoscopic adrenal surgery for neuroblastoma in children. J Urol 2003; 170: 932-5.

7. Lecleir MD, De Lagausie P, Becmeur F, et al. Laparoscopic resection of abdominal neuroblastoma. Ann of Surg Oncol 2008; 15 : 117-24.

8. Skarsgard ED, Albanese CT. The safety and efficacy of laparoscopic adrenalectomy in children. Arch Surg 2005; 140: 905-8.

9. Myśliwiec P, Dadan J, Łukaszewicz J, Sulik M. Two tumours of the right adrenal gland treated videoscopically by the posterior retroperitoneal approach. Videosurgery and other miniinvasive techniques 2009; 4: 126-30.

10. Chow A, Aziz O, Purkayastha S, et al. Single incision laparoscopic surgery for acute appendicitis: feasibility in pediatric patients. Diagn Ther Endosc 2010; 2010: 2949-58.

11. Dutta S. Early experience with single incision laparoscopic surgery: eliminating the scar from abdominal operations. J Pediatric Surg 2009; 44: 1741-5. 